\title{
BeMonitored: Monitoring psychophysiology and behavior using Android in phobias
}

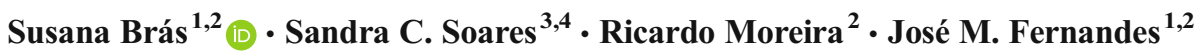

Published online: 15 July 2015

(C) Psychonomic Society, Inc. 2015

\begin{abstract}
It is of the utmost importance that researchers can recreate, as accurately as possible, real-life conditions in psychological studies. However, that is not always possible. Given that phobias are rather context-specific, their study is the ideal candidate to assess the feasibility of using a mobile and wearable device for obtaining physiological and behavioral data. In this article, we propose BeMonitored, a smartphone-based solution to support more ecologically valid monitoring of psychological experiments. BeMonitored delivers customizable, specific context-dependent audiovisual stimuli and uses external resources connected via Bluetooth or a smartphone's own resources, while capturing the participant's behavior, physiology, and environment. We used BeMonitored in a spider phobia case study and showed that spider phobics differed from control participants in face motion, captured by the smartphone camera. Moreover, our results also revealed heart rate differences between spider and neutral stimuli in phobic participants. The presented results emphasize the usefulness of smartphones for phobia monitoring. Considering their intrinsic characteristics, smartphones may
\end{abstract}

Susana Brás

susana.bras@ua.pt

1 Instituto de Engenharia Electrónica e Informática de Aveiro (IEETA), Universidade de Aveiro, Campus Universitário de Santiago, 3810-193 Aveiro, Portugal

2 Departamento de Electrónica, Telecomunicações e Informática, Universidade de Aveiro, Aveiro, Portugal

3 Center for Health Technology and Services Research (CINTESIS), Department of Education, University of Aveiro, Aveiro, Portugal

4 Department of Clinical Neurosciences, Division of Psychology, Karolinska Institute, Stockholm, Sweden constitute the natural evolution from the lab to more realistic contexts.

Keywords Psychophysiology · Behavior · Spider phobia . Online monitoring $\cdot$ Mobile device

Specific phobias are common mental disorders and cause a substantial clinical burden (LeBeau et al., 2010). Spider phobia, in particular, is amongst the most prevalent forms of specific phobia (Agras, Sylvester, \& Oliveau, 1969) and involves persistent and intense fear of spiders, as well as avoidance behavior regarding every context in which a confrontation with spiders is anticipated (American Psychiatric Association, 2013). Although animal phobia may be considered as having limited clinical relevance, this circumscribed anxiety disorder (Soares, Esteves, \& Lundqvist, 2015; Soares, Esteves, Lundqvist, \& Öhman, 2009) offers a valuable scientific model for investigating psychophysiological reactions (e.g., heart rate) and behavioral changes (e.g., avoidance) (for reviews, see Öhman \& Mineka, 2001; Öhman, Soares, Juth, Lindström, \& Esteves, 2012).

One of the central tenets of several cognitive-behavioral models of anxiety disorders is to attribute avoidance behavior as a maintenance factor of anxiety (e.g., Beck, Emery, \& Greenberg, 1985). Avoidance either can be reflected in a complete refusal to confront the phobic stimulus (e.g., a spider in the case of spider phobics) or can involve safety behaviors, such as head movements away from such stimuli. Thus, observing behavior is highly informative for both the diagnosis and assessment of treatment efficacy. The behavioral approach/avoidance test (BAT) is one of the most common behavioral observation methods in specific phobia (Antony \& Barlow, 2011). BAT involves the confrontation of the 
phobic individuals with their feared object while several aspects of behavior (overt and covert) are being measured (e.g., proximity to the object and psychophysiological responses) (Hood \& Antony, 2012).

Although capturing and quantifying physiology and behavior in realistic environments is very relevant in psychological science (Lang, Greenwald, Bradley, \& Hamm, 1993), most studies have been performed in highly controlled settings, thus neglecting the relevance of the context and environment in the studied phenomena. The benefits of laboratory studies are undeniably relevant, but they do not portray real-life scenarios. However, with the advent of both wearable and mobile devices, some of the main technical difficulties found outside the laboratory (i.e., logistics, lack of mobile setups) can be surpassed and new scenarios for data gathering outside the lab considered, allowing a quantifiable and realistic-context follow-up.

Recent technological developments in the areas of biomedical sensors, mobile devices, and wireless telecommunications allow new solutions to measure, communicate, and process information in real time and anywhere (Brás, Fernandes, \& Cunha, 2013; Colunas, Fernandes, Oliveira, \& Cunha, 2011; Ribeiro, Colunas, Marques, Fernandes, \& Cunha, 2011). Good examples are mobile devices, in particular the smartphones that are becoming a ubiquitous resource, with a huge potential in the psychology field. Yet, validation studies are still lacking (Bless, Westerhausen, Kompus, Gudmundsen, \& Hugdahl, 2014; Miller, 2012). Smartphones merge several important properties that have great potential: their cost, when compared to typical laboratory settings (see, e.g., Lee et al., 2012); their availability, allowing a better coverage of the target population; and their adaptability to the environment - to context and participants' behaviors (through targeted application development) - thus providing valuable feedback and assessment, for instance, as a treatment outcome. They can also act as a stimulus delivery system - namely through static images, video, and audio - while relying on sensors to collect precise, objective, and ecologically valid data from real settings (e.g., motion, location, and psychophysiology). A few recent studies have already addressed the validity of technology use for assessments of psychological states (Andersson et al., 2009; Botella, Bretón-López, Quero, Baños, \& García-Palacios, 2010; Botella et al., 2011; Picard \& Liu, 2007; Stopczynski et al., 2014), and in particular of mobile systems as a portable and inexpensive solution (Botella et al., 2010, 2011; Picard \& Liu, 2007; Stopczynski et al., 2014). However, studies investigating the validity of monitoring devices including psychophysiological signals, such as electrocardiography (ECG), are still very much in its infancy.

In this article, we propose BeMonitored, a system supported on a mobile device. BeMonitored intends to explore the use of mobile and wearable systems in the assessment and study of psychological disorders outside the lab. BeMonitored can deliver context-aware stimuli (e.g., time, GPS location) based on both audio and video, while monitoring both behavior (e.g., face motion and activity using the accelerometer) and physiological variables (e.g., ECG, as in the present configuration using an external wearable device; Ribeiro et al., 2011).

As a proof of concept for BeMonitored, we focused on phobias - more specifically, spider phobia. As has been reported in the literature, the exposure to a phobic stimulus by a phobic person triggers an avoidance response (e.g., Öst, 1985). Since it is triggered by very clear and specific ecological stimuli, it is the ideal type of disorder to study the impact of using a mobile monitoring solution. Mobile monitoring could provide solutions to quantify the reaction to phobic stimuli in the assessment of the phobia level or in the treatment follow-up-all in a more realistic and ecological context. Participants were monitored and physiological and behavioral variables were collected, such as ECG, face tracking, video, and audio (i.e., in a multimodal approach), in an indoor setup in order to evaluate the value and reliability of smartphones as a means to monitor phobia.

\section{Materials and method}

\section{Participants}

The study involved a group of 30 university students at the University of Aveiro, Portugal, who volunteered to participate. All participants filled out the Fear of Spiders Questionnaire (Klorman et al., 1974). On the basis of the questionnaire scores, we assumed that individuals with scores above the 75th percentile were potentially phobic, and those scoring below 25th percentile were considered as a control, nonphobic group. The participants' group score was $13 \pm 8$ (mean \pm standard deviation), the score for the 75 th percentile was 20 , and that of the 25 th percentile was 7 . From the initially 30 individuals who filled out the questionnaire, only seven (two males and five females) fulfilled the inclusion criteria or were available to perform the test- four potential phobics (f2, f5, f6, and f7) and three controls (f1, $\mathrm{f} 3$, and $\mathrm{f} 4$ ). The selection procedure to create the high-fear groups resulted in participants with fear levels in the phobic rank, but did not include a formal DSM-V diagnosis of animal phobia (American Psychiatric Association, 2013). The participants had normal or corrected-to-normal vision, did not suffer from any mental or neurological disorder, and were medication free.

The study was approved by the Ethics Committee of the University of Aveiro, Portugal, and both the guidelines of the Declaration of Helsinki and the standards of the American Psychological Association were followed. Participation in the experiment was based on written informed consent, 
including the right to end participation at any time. Participants were rewarded with course credits.

\section{Experimental setup and protocol}

During the data collection, the smartphone was placed in front of the user, in order to acquire video frames of the participant's face, with the smartphone's front-facing camera, while presenting video stimuli (Fig. 1). To avoid setup configuration problems and ensure that the participant's face was detected regardless of height, the distance to the device, or even the context in which the device was used, the BeMonitored application was developed to include a calibration process to ensure the individual's correct position. The user-worn ECG electrodes and a photopletismography (PPG) sensor in order to acquire physiological data. During the calibration phase, the system also extracted the initial heart rate.

A video was presented in each session, during which head/eye movements and heart rate were monitored. The volunteers were exposed to a total of $150 \mathrm{~s}$ of video: $10 \mathrm{~s}$ with a textual sequence, $35 \mathrm{~s}$ with the controll segment, $70 \mathrm{~s}$ with spiders, and $35 \mathrm{~s}$ with the control 2 segment. The setup used is depicted in Fig. 1.

For each volunteer dataset, we split the data into three groups: controll (first neutral video), spider (video containing spiders: active), and control 2 (second neutral video). The dependent variables were the heart rate, head movement in the $x$ and $y$ axes, and the fixational stability of head movement. The last variable was calculated as

$\mathrm{P}(\mathrm{t})=\mathrm{x}_{-} \operatorname{dist}^{2}(\mathrm{t})+\mathrm{y}_{-} \operatorname{dist}^{2}(\mathrm{t}), \mathrm{t}=1, \ldots, \mathrm{N}$,

where $\mathrm{x}$ _dist (y_dist) was the distance from the current midpoint eyes position in the $x(y)$ axis to the baseline eyes position, and $N$ was the length of the data collection record.
The segments were compared within each volunteer dataset (paired statistical analysis), using a Wilcoxon signed-rank test (comparing the segments' medians), and also using an $F$ test (comparing the segments' variances). We considered $p<.05$ as marking the significance level. A Bonferroni correction was implemented for multicomparison analysis.

\section{BeMonitored architecture}

The BeMonitored system has two main logical blocks - the acquisition box and the smartphone, depicted in Fig. 2. The acquisition box is responsible for the acquisition of physiological information collected from the human participants (1). The smartphone runs the BeMonitored application, which receives the incoming information in the signal acquisition module (2). This module updates the data display and stores the raw data. The signal processing unit (3) is responsible for processing the incoming signals from the acquisition box (1) and performs the relevant event detection. BeMonitored relies on the multimedia resources of the smartphone to acquire both audio and videoimage information using the smartphone cameras, and later the video data are processed by the image processing unit (4).

The main user interface is provided by the BeMonitored application responsible for coordinating the several modules during synchronous data acquisition and storage. To ensure that all acquired and processed data are synchronized, all data are archived jointly with a timestamp in the memory store (5). A .txt format file is used to simplify data exporting and human readability. Besides allowing online monitoring of the main information in the smartphone display (6) during acquisition, BeMonitored (when not acquiring a session) also provides basic interfaces for session management, review, and exporting.
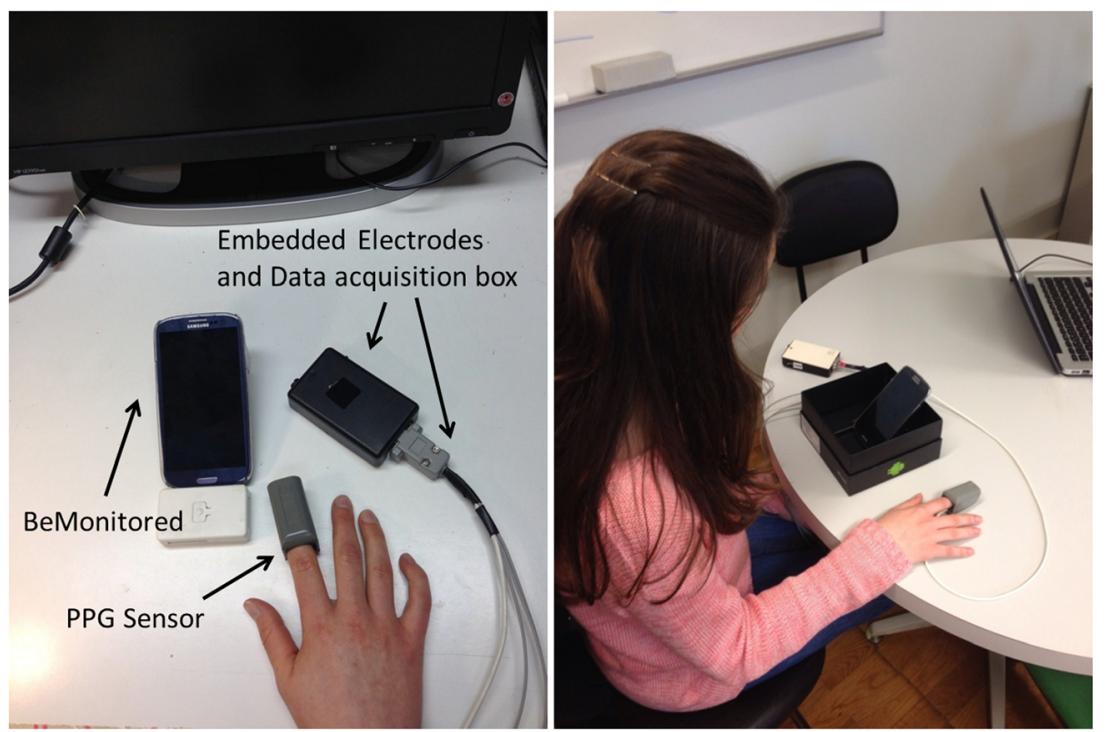

Fig. 1 BeMonitored: Components and sample use during a session in a spider phobia study. Note that the participants faced the smartphone but did not hold it, in order to avoid damage to the equipment due to extreme responses of the phobic person 


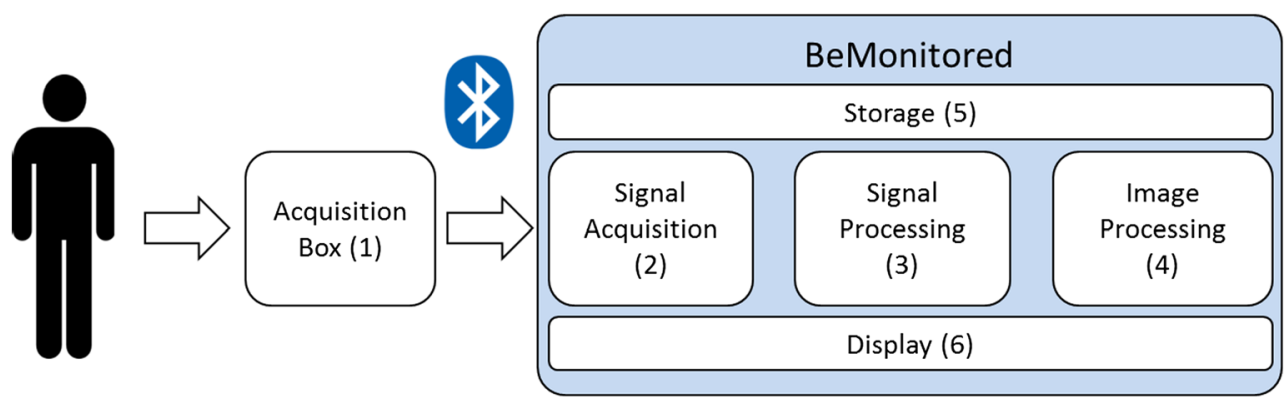

\section{a}

Fig. 2 BeMonitored block diagram: The main components are the acquisition box (a) and BeMonitored, running on a smartphone (b). BeMonitored, besides supporting the acquisition and processing of data

\section{BeMonitored system}

The implementation of BeMonitored is based on the Android OS, and a Samsung Galaxy S3 phone was used as the main deployment smartphone. We used the wearable RTABP system (Ribeiro et al., 2011) to acquire ECG and PPG at a 500-Hz sampling frequency, transmitted to the smartphone using a Bluetooth connection. Although this equipment was developed in house, the system is prepared to collect ECG signals from other equipment.

Once parsed from RTABP, the ECG data are processed using an implementation of the QRS detection algorithm by Hamilton/Pan-Tompkins (Hamilton \& Tompkins, 1986; Pan $\&$ Tompkins, 1985) to calculate the heart rate (HR). This algorithm has already been implemented and tested in the DroidJacket system (Colunas et al., 2011). On the basis of HR, we performed a detector for tachycardia events that triggered an alarm whenever the HR values were higher than $100 \mathrm{bpm}$ (Timby, 2009). The detection of the event triggered an intent at Android OS level that generated an audiovisual alarm, translated in the device vibration with a distinct pattern.

\section{Behavior: Face tracking, audio, and video}

To monitor behavior, we relied on the video and audio capabilities of the smartphone. BeMonitored simultaneously collects, processes, and stores both audio (environment sound and voice) and video from the phone's front-facing camera. To accomplish the online video processing, we opted to acquire sequences of individual frames instead of a video stream. Although the sampling frequency is lower (three frames per second), it allows for processing online and full tracking of the face motion on the basis of the extraction of the eyes position for each acquired frame.

Each captured frame is preprocessed: flipped (rotation of $270 \mathrm{deg}$ ) and subsampled to a power-of-two size (currently to 24 ), which returns an image that is $1 / 4$ the width/height of the original with $1 / 16$ the number of pixels, to optimize the during the monitoring session, also supports basic session management, review, and exporting functionalities

memory footprint and processing time. The face is detected using the Android OS's native face detection algorithm, also allowing the identification of the eye position (whenever possible). The middle point between the two eyes is used to estimate the position of the eyes, which is used as our facetracking solution (Fig. 3).

BeMonitored also creates a record for each participant and session containing personal (photo, name, e-mail) and session-specific information (date, duration of the experimental session) (see Fig. 4a). The record includes all calculated parameters, namely pictures taken, faces detected or not detected, average, higher and lower HR, and events (e.g., eyes midpoints and HR), with the respective timestamps (lower and higher values) (Fig. 4d). This information is stored in an internal application database using SQLite, which can be accessed and analyzed later.

All acquired data (video frames captured, the audio file, and physiological data streams) are stored on the device memory. A file for each session is created with the processed data (eyes midpoints and HR values).

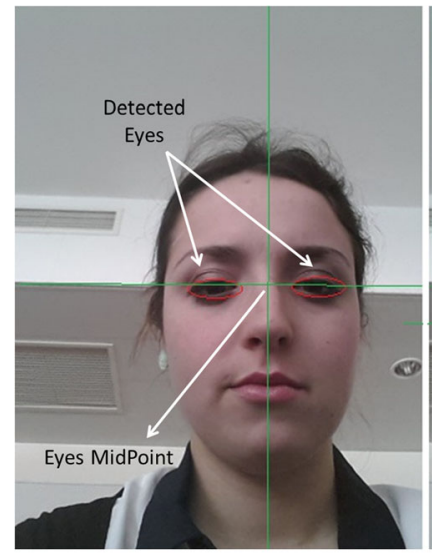

a

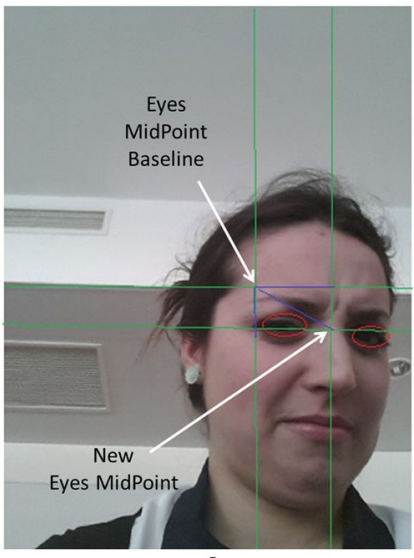

b
Fig. 3 Face-tracking example using eye midpoint estimation. Using an initial position (a), the face tracker estimates the face displacement during the session (b) 


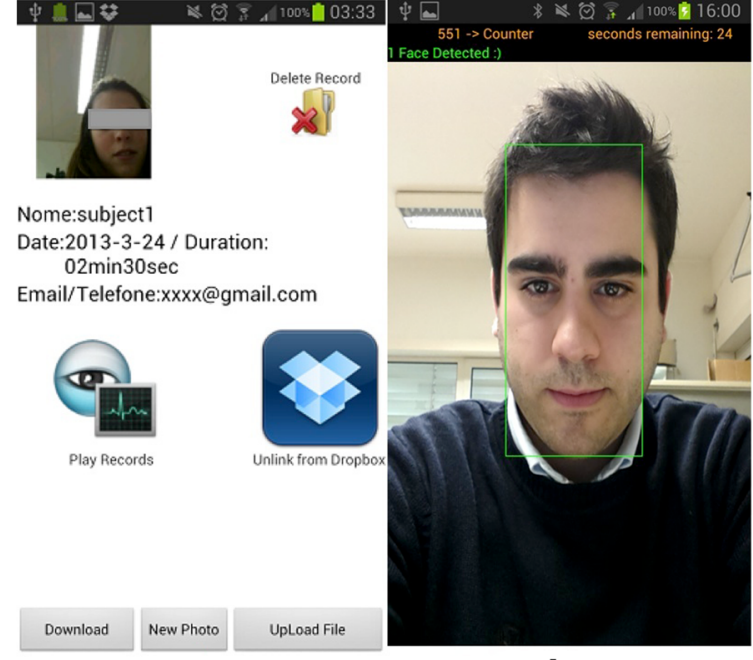

a

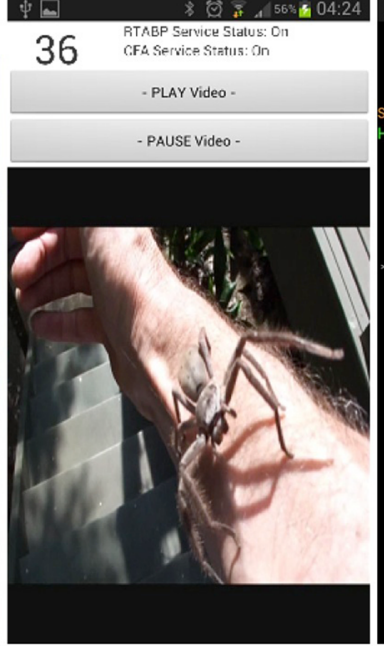

C

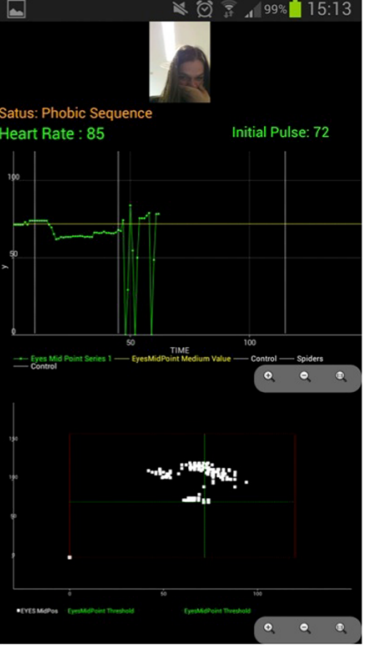

d

Fig. 4 BeMonitored user interface. Here are some representative snapshots of the BeMonitored user's interface: (a) record management, including session review and data export; (b) video calibration screen, to ensure that the participant is in front of the smartphone; (c) snapshot from the spider segment video; (d) online/offline review screen, presenting the

\section{Data acquisition}

Each session is defined by a specific video (the stimulus) and a set of video-dependent timestamps (presented in the online display, Fig. 4c) in order to tag relevant events or protocol transitions. This description is contained in an uploaded XML file and can be customized according to the aim of the session.

During a BeMonitored session, there are three main stages: calibration, baseline, and the session protocol. The calibration process is performed at the start of every acquisition. At this stage, an online face-tracking process starts, using the phone's

participant's face on top, the initial and current heart rate (72 and 84, respectively) and a graph of the face position during the session. Vertical lines represent two protocol condition changes. On the bottom is a chart presenting the face motion across time

front camera. The user sees him-/herself on the screen, and with the help of a feedback rectangle, he/she adjusts the position of the head. This procedure ensures that the system is able to capture the volunteer's face (Fig. 4b). During the calibration phase, the system starts the physiological (currently ECG) data collection. Overall, this protocol stage lasts $1 \mathrm{~min}$ and establishes the baseline for the HR and the head/eye position measurements.

In order to establish a baseline for future analysis, the first $10 \mathrm{~s}$ of the video stream are presented to the user with a textual sequence. During this period, the system makes an average of
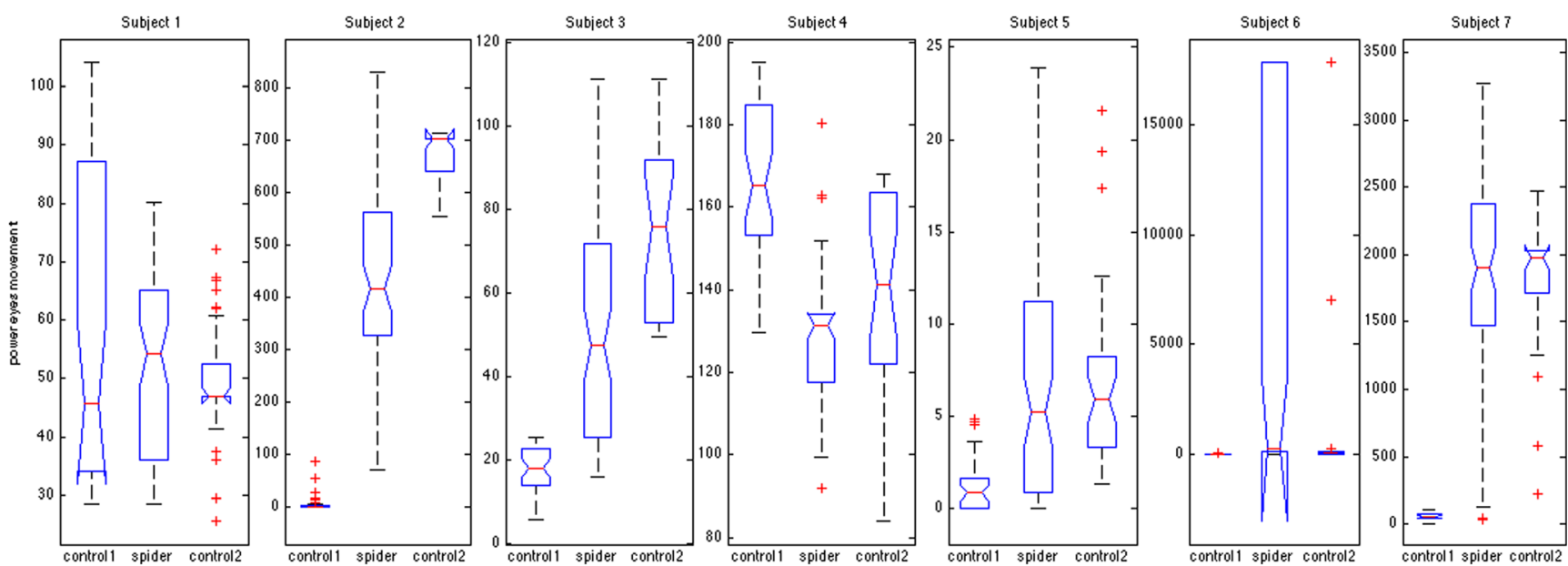

Fig. 5 Dispersion of face-tracking movement (fixational stability in relation to initial position) for the seven volunteers, considering the control1, spider exposure, and control 2 segments. The phobic participants are Subjects 2, 5, 6, and 7. Subject 6 is an extreme example; during the session, he/she felt high discomfort and tried not to

focus on the video in the spider segment, with that response being translated to a high variability in spider segment. Each boxplot represents the $75 \%$ percentile, median, and $25 \%$ percentile for the face-tracking movement 
Table 1 Medians and percentiles 25 and 75 are presented in the table for all participants, in the format median [percentile25, percentile75]

\begin{tabular}{llll}
\hline & control1 & spider & control2 \\
\hline f1 & $83.00[81.00,83.27]$ & $81.00[78.00,83.00]$ & $83.54[83.00,85.00]$ \\
f2 & $72.30[65.94,80.01]$ & $78.95[73.63,83.33]$ & $72.30[68.18,77.94]$ \\
f3 & $125.00[122.40,128.30]$ & $109.72[96.00,113.86]$ & $93.00[91.71,95.82]$ \\
f4 & $125.00[125.00,127.58]$ & $96.00[84.26,120.00]$ & $81.84[79.38,82.76]$ \\
f5 & $57.00[57.00,57.72]$ & $57.00[56.00,57.60]$ & $56.00[56.00,57.00]$ \\
f6 & $93.75[88.24,99.85]$ & $97.09[86.52,108.23]$ & $95.55[89.55,105.63]$ \\
f7 & $71.00[69.00,73.00]$ & $78.00[73.00,85.00]$ & $66.00[65.00,68.00]$ \\
\hline
\end{tabular}

the first 30 midpoints of the eyes coordinated to get the baseline ( 3 frames $/ \mathrm{s}=30$ shots) for the initial face position.

After the calibration and baseline stages, the video starts to be displayed and the behavior (face position) and physiological data to be acquired. During this stage, the acquired data are used to extract the online signal and video-related parameters. The test and the acquisition process end when the video also ends.

\section{Data archiving and exporting}

Besides storing data in the Android file system, we also decided to enable export ability of the data to the cloud to a common, online folder, to serve as an offline storage/ analysis resource. Currently, we are using Dropbox and have deferred to it all the data transfer and synchronization, to distance both the application and the user from the data transfer process. The export process implies an explicit export option from the BeMonitored user. The selection of Dropbox was based on the good native support for both Android and other major OSs.

a

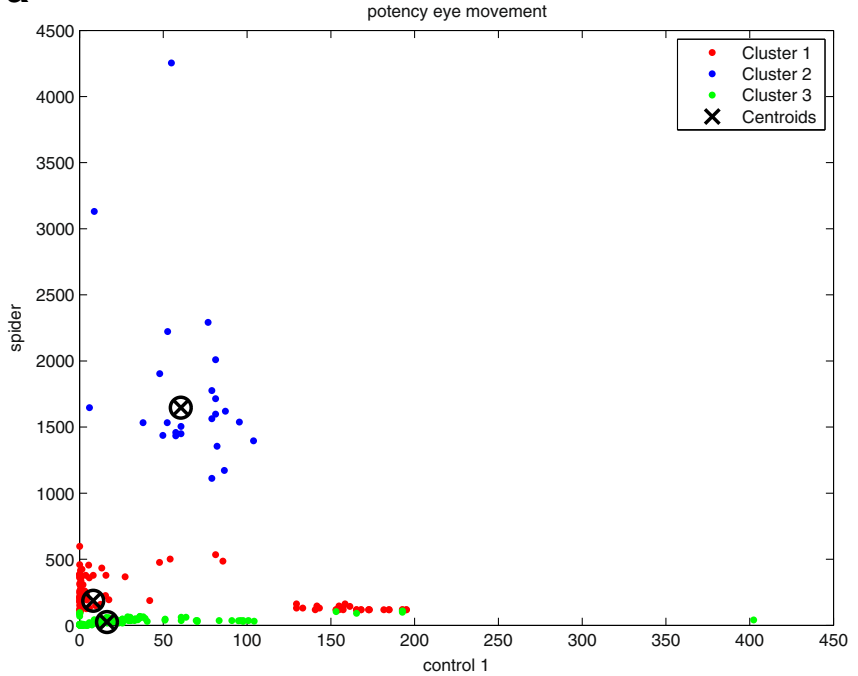

\section{Results and discussion}

This article's major goal is to present the BeMonitored software as a new tool for behavioral and physiology data collection in ambulatory conditions. To accomplish this goal, we collected and analyzed data (fixational stability and HR) in order to support the hypothesis that presenting the fear stimulus on a smartphone induces both behavioral and physiological alterations in the participant.

The data acquired and processed by the monitoring application revealed an alteration in the behavior of all volunteers while they were exposed to spiders. The fixational stability was significantly different between the control1 and control 2 segments $(p<.05)$, except in one nonphobic volunteer (f1). When comparing the spider with the controll segment, a similar result was obtained - significant differences $(p s<.05)$ for all volunteers except one nonphobic subject (f1), indicating a behavioral response change when spiders were shown. A significant difference $(p<.05)$ was also observed between the spider and control 2 segments, in all except three (f1 [nonphobic] and f6 and $\mathrm{f} 7$ [phobic]) volunteers. Our results also showed that the variance of the fixational stability in the spider segment

b

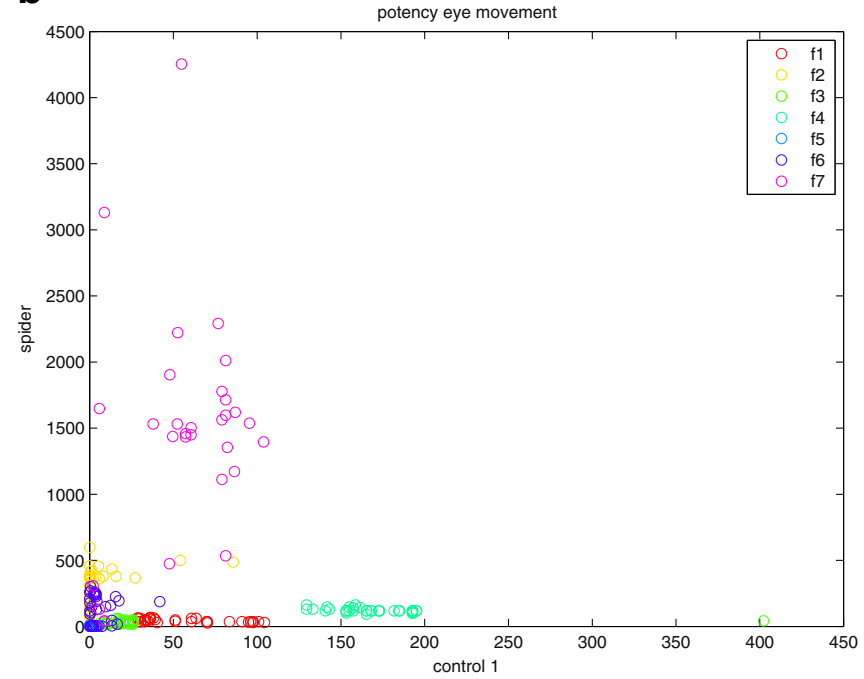

Fig. 6 (a) Clustering using the K-Medians method, relating the controll and spider segments; (b) dispersion diagram of the data of each participant 
a

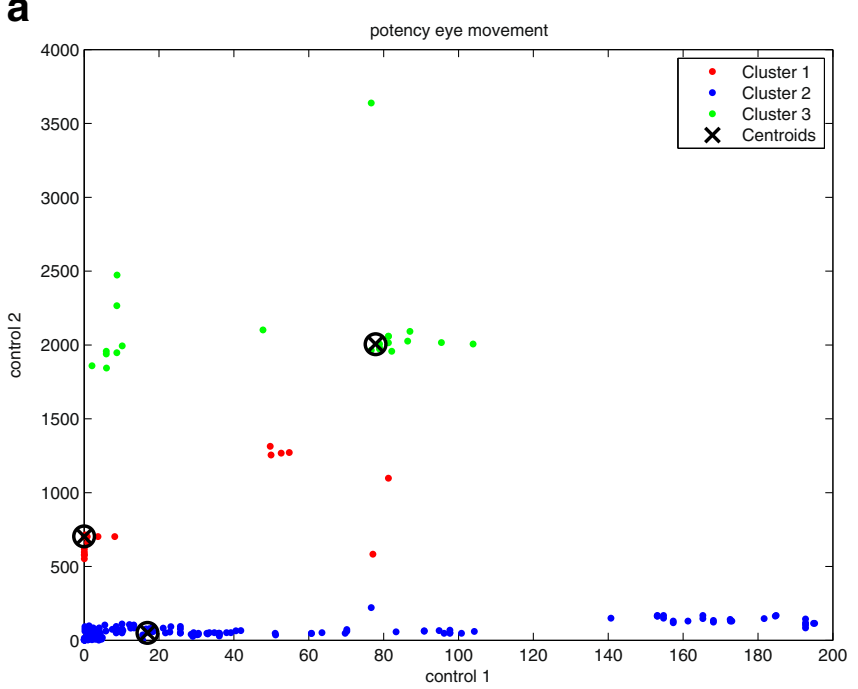

b

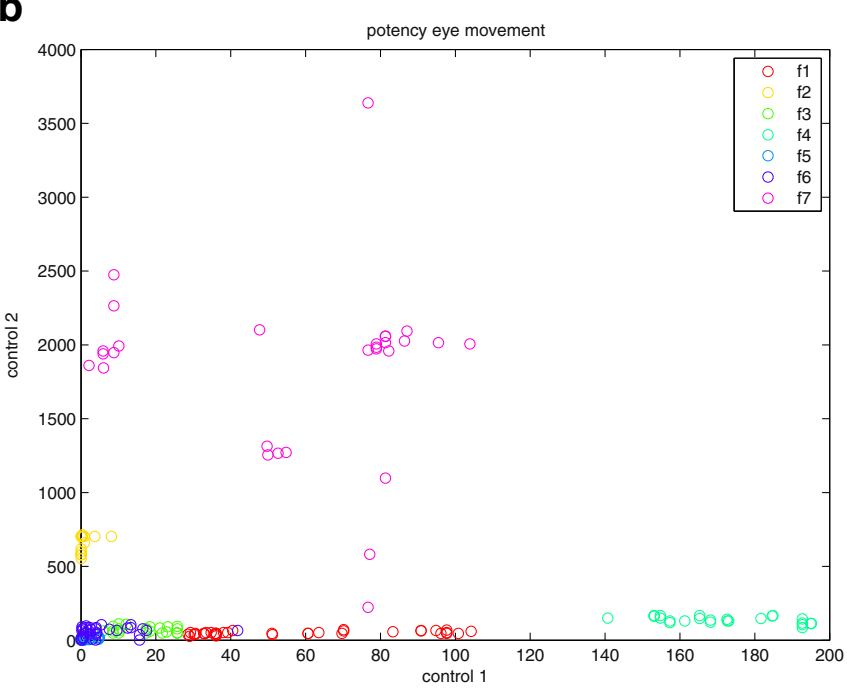

Fig. 7 (a) Clustering using the K-Medians method, relating the control1 and control2 segments; (b) dispersion diagram of the data of each participant

was higher than the variance in the control 2 segment $(p<.05)$, except in the nonphobic volunteers (f1, f3, and f4) and $\mathrm{f} 7$ (the boxplots in Fig. 5 allow a similar interpretation).

Regarding the HR responses (Table 1), we found a significant difference between the two control segments except in two phobic volunteers ( $\mathrm{f} 2$ and $\mathrm{f5}, p \mathrm{~s}<.05$ ). A significant difference was apparent in almost all volunteers $(p<.05$; except in $\mathrm{f5}$ and $\mathrm{f} 7$ [phobic]) when comparing the control1 and spider segments. This may have been motivated by the fact that these participants were aware that they would be confronted with spiders, which might have altered their baseline anxiety (e.g., HR values). The observed variance in the spider segment was higher than the variance in the controll segment (i.e., there was an activation, $p<.05$ ), in all except two phobic participants (f2 and f7). Moreover, the increase of variance in HR and in fixational stability suggested that

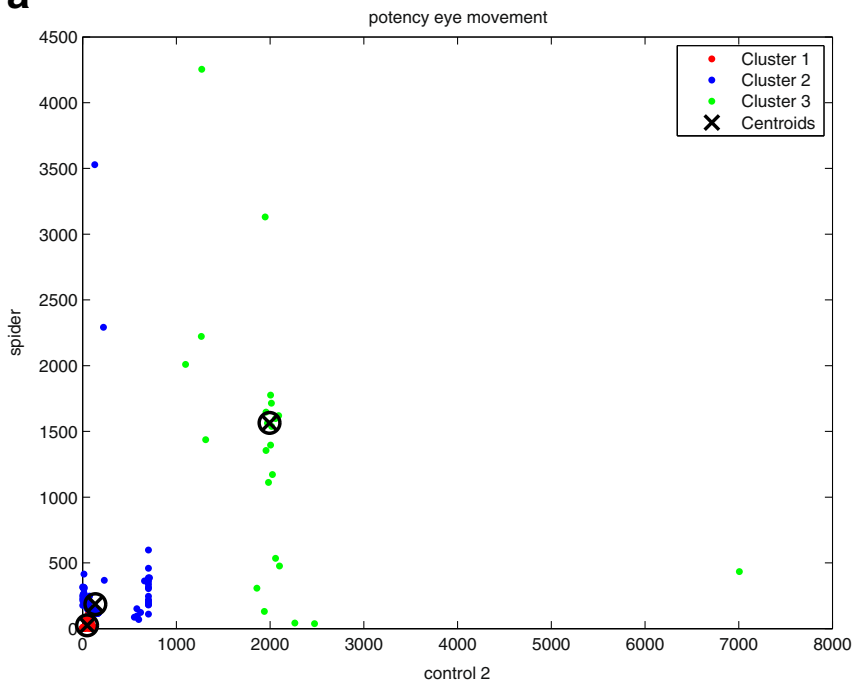

individuals displayed a specific response, both psychological and behavioral, after the exposure to spiders.

The clustering algorithm K-Medians was used to study the relation between the data segments and dispersion diagrams. Initially, the data were analyzed using a configuration with two clusters $(K=2)$, in order to identify the two groups (nonphobic and phobic). However, by using this configuration, one participant constituted a cluster, which was justified by his/her extreme reaction to the stimulus. Therefore, and since we intended to separate at least the nonphobic participants, we decided to include an extra cluster, in order to group participants with the same characteristics. Consequently, we used a configuration with three clusters $(K=3)$, thus grouping nonphobic, probable phobic, and extreme reactions. The three clusters identified (Cluster 1, Cluster 2, and Cluster 3) are represented in the Figs. 6a, 7a, and 8a.

b

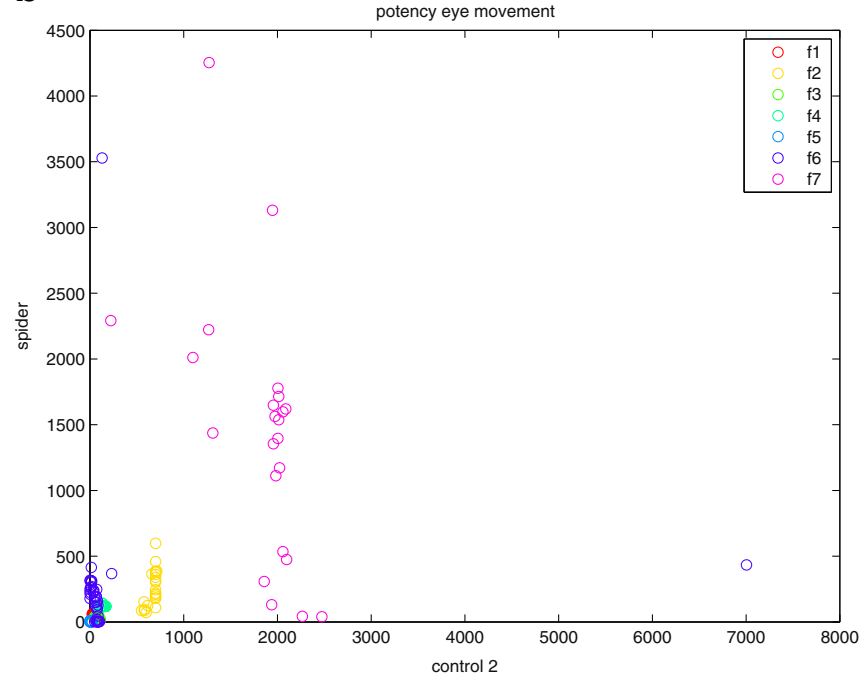

Fig. 8 (a) Clustering using the K-Medians method, relating the control2 and spider segments; (b) dispersion diagram of the data of each participant 
The analysis of the clusters suggested a possible discrimination between the phobic and nonphobic groups. Considering controll versus spider (Fig. 6a and b), Cluster 2 contained data from $\mathrm{f} 7$ (phobic), Cluster 1 data from $\mathrm{f} 2$ and $\mathrm{f} 6$ (both phobic), and Cluster 3 contained the remaining data (phobic and nonphobic).

In relation to control1 versus control2 (Fig. $7 \mathrm{a}$ and b), Cluster 3 contained the data from $\mathrm{f} 7$ (phobic); Cluster 1 contained data from $\mathrm{f} 2$ and some from $\mathrm{f} 7$ (both phobic); and Cluster 2 contained the remaining data (mixing phobic and nonphobic data). Finally, in control2 versus spider (Fig. 8a and b), Cluster 2 contained data from $\mathrm{f} 2, \mathrm{f} 4$, and $\mathrm{f6}$ ( $\mathrm{f} 4$ is nonphobic); Cluster 3 contained data from $\mathrm{f} 7$ (phobic); and Cluster 1 was composed of the remaining participants' data (collapsing phobic and nonphobic).

By analyzing Figs. 6a, 7a, and 8a, it was evident that participant $\mathrm{f} 7$ was always differentiated from the other volunteers, representing in some cases an independent cluster (Figs. 6 and 8).

Overall, even considering the limited number of participants, the results suggest that by using these clusters it might be possible to distinguish spider-phobic from control participants using the fixational stability captured by the smartphone's camera. One possible justification for the obtained results may be that, for control participants, the spider stimuli were evaluated as innocuous, and therefore did not significantly influence their behavioral and physiological responses. On the other hand, the phobic participants showed a distinct behavioral response, which was consistent with the extensive literature on fear and phobias (e.g., Öhman \& Mineka, 2001). In fact, the fear or the discomfort experienced by those individuals when confronted with the stimuli were translated into an avoidance response, as has been mentioned in the literature (Antony \& Barlow, 2011; Hood \& Antony, 2012; Wilhelm \& Grossman, 2010).

The sample size constitutes a limitation in the extrapolation of the results, since it is not representative of a phobic population. The overall pattern of results from the present study shows the potential usefulness of smartphones in phobia monitoring, regardless of the small sample size. Due to the inherent characteristics of smartphones, they may indeed constitute a potent instrument that may enable a natural evolution from lab monitoring to outside monitoring of several disorders. However, the evolution from the indoor to the outdoor setup will only be possible after ensuring smartphones' physical integrity in the face of extreme reactions during the exposure to phobic stimuli.

\section{Conclusion}

In this article, we proposed BeMonitored, an affordable smartphone-based solution to support more ecologically valid monitoring of psychological experiments. BeMonitored delivers customizable, specific, context-dependent audiovisual stimuli and is able to capture the participant's behavior, physiology, and environment by using external resources connected via Bluetooth or the smartphone's camera and audio. BeMonitored produces synchronous data for each session in an accessible and readable text format. Although BeMonitored was initially intended to be fully autonomous, we extended it with the ability to explicitly export the data from the application directly to the cloud using Dropbox (e.g., a Research folder on the desktop). Currently, we are proceeding with validation of BeMonitored to release it as a freely available support tool. We aim to provide an information system back office for data management, as well. BeMonitored also provides basic session management, namely for demographics, and supports offline review of all related information for a given session (acquired data and participants' demographics).

We found significant variations of HR during the spider events and showed that spider phobics could be differentiated from control participants using solely the face motion captured by the smartphone camera. Although further tests are needed, due to the limited number of participants, BeMonitored has proven to be a viable system that by design can be further extended to provide other physiological and behavioral parameters.

Although our focus was on spider phobia, BeMonitored could be applied to other phobia-related assessment and monitoring, especially in cognitive-behavioral therapy scenarios (e.g., Öst, 1985).

Author note This work was supported by the European Regional Development Fund (FEDER) through the COMPETE program, and by the Portuguese Government through the Fundação para a Ciência e a Tecnologia (FCT), in the scope of the projects Incentivo/EEI/ UI0127/2014 and UID/CEC/00127/2013 (Instituto de Engenharia Electrónica e Informática de Aveiro, IEETA/UA, www.ieeta.pt), PTDC/EEI-ELC/2760/2012 (VitalResponder 2.0), CMUP-ERI/FIA/0031/ 2013 (VR2Market, by the CMU-Portugal program), PTDC/NEU-SCC/ 0767/2012 (ASD), and Cloud Thinking (CENTRO-07-ST24-FEDER002031, co-funded by the QREN, "Mais Centro" program). S. Brás acknowledges a postdoc grant from FCT, reference number SFRH/BPD/ 92342/2013.

\section{References}

Agras, W. S., Sylvester, D., \& Oliveau, D. (1969). The epidemiology of common fear and phobia. Comprehensive Psychiatry, 10, 151-156.

American Psychiatric Association. (2013). Diagnostic and statistical manual of mental disorders (5th ed., text rev.). Washington, DC: Author.

Andersson, G., Waara, J., Jonsson, U., Malmaeus, F., Carlbring, P., \& Ost, L.-G. (2009). Internet-based self-help versus one-session exposure in the treatment of spider phobia: A randomized controlled trial. Cognitive Behaviour Therapy, 38, 114-120. doi:10.1080/ 16506070902931326 
Antony, M. M., \& Barlow, D. H. (2011). Handbook of assessment and treatment planning for psychological disorders (2nd ed.). New York, NY: Guilford Press.

Beck, A. T., Emery, G., \& Greenberg, R. L. (1985). Anxiety disorders and phobias. New York, NY: Basic Books.

Bless, J. J., Westerhausen, R., Kompus, K., Gudmundsen, M., \& Hugdahl, K. (2014). Self-supervised, mobile-application based cognitive training of auditory attention: A behavioral and fMRI evaluation. Internet Interventions, 1, 102-110.

Botella, C., Breton-López, J., Quero, S., Baños, R. M., García-Palacios, A., Zaragoza, I., \& Alcaniz, M. (2011). Treating cockroach phobia using a serious game on a mobile phone and augmented reality exposure: A single case study. Computers in Human Behavior, 27, 217-227.

Botella, C., Bretón-López, J., Quero, S., Baños, R., \& García-Palacios, A. (2010). Treating cockroach phobia with augmented reality. Behavior Therapy, 41, 401-413.

Brás, S., Fernandes, J. M., \& Cunha, J. P. S. (2013). ECG delineation and morphological analysis for firefighters tasks differentiation. In P. R. Rodrigues et al. (Eds.), Proceedings of CBMS 2013-26th IEEE International Symposium on Computer-Based Medical Systems (pp. 516-517). Piscataway, NJ: IEEE Press.

Colunas, M. M. F., Fernandes, J. M. A., Oliveira, I. C., \& Cunha, J. P. S. (2011). Droid jacket: A mobile monitoring system for a team. In Proceedings of 7th International Wireless Communications and Mobile Computing Conference, IWCMC (pp. 2157-2161). Piscataway, NJ: IEEE Press.

Hamilton, P. S., \& Tompkins, W. J. (1986). Quantitative investigation of QRS detection rules using the MIT/BIH arrhythmia database. IEEE Transactions on Biomedical Engineering BME, 33, 1157-1165.

Hood, H. K., \& Antony, M. M. (2012). Evidence-based assessment and treatment of specific phobias in adults. In T. E. Davis III, T. H. Ollendick, \& L.-G. Öst (Eds.), Intensive one-session treatment of specific phobias (pp. 19-42). New York, NY: Springer.

Klorman, R., Weerts, T. C., Hastings, J. E., Melamed, B. G., \& Lang, P. (1974). Psychometric description of some specific-fear questionnaires. Behavior Therapy, 5, 401-409.

Lang, P. J., Greenwald, M. K., Bradley, M. M., \& Hamm, A. O. (1993). Looking at pictures: Affective, facial, visceral, and behavioral reactions. Psychophysiology, 30, 261-273. doi:10.1111/j.1469-8986. 1993.tb03352.x

LeBeau, R. T., Glenn, D., Liao, B., Wittchen, H.-U., Beesdo-Baum, K., Ollendick, T., \& Craske, M. G. (2010). Specific phobia: A review of DSM-IV specific phobia and preliminary recommendations for DSM-V. Depression and Anxiety, 27, 148-167. doi: $10.1002 /$ da. 20655
Lee, H., Baniqued, P. L., Cosman, J., Mullen, S., McAuley, E., Severson, J., \& Kramer, A. F. (2012). Examining cognitive function across the lifespan using a mobile application. Computers in Human Behavior, 28, 1934-1946.

Miller, G. (2012). The smartphone psychology manifesto. Perspectives on Psychological Science, 7, 221-237. doi:10.1177/1745691612441215

Öhman, A., \& Mineka, S. (2001). Fears, phobias, and preparedness: Toward an evolved module of fear and fear learning. Psychological Review, 108, 483-522. doi:10.1037/0033-295X.108.3.483

Öhman, A., Soares, S. C., Juth, P., Lindström, B., \& Esteves, F. (2012). Evolutionary derived modulations of attention to two common fear stimuli: Serpents and hostile humans. Journal of Cognitive Psychology, 24, 17-32.

Öst, L.-G. (1985). Coping techniques in the treatment of anxiety disorders: Two controlled case studies. Behavioural Psychotherapy, 13, 154-161.

Pan, J., \& Tompkins, W. J. (1985). A real-time QRS detection algorithm. IEEE Transactions on Biomedical Engineering BME, 32, 230-236.

Picard, R. W., \& Liu, K. K. (2007). Relative subjective count and assessment of interruptive technologies applied to mobile monitoring of stress. International Journal of Human-Computer Studies, 65, 361375. doi:10.1016/j.ijhcs.2006.11.019

Ribeiro, D. M. D., Colunas, M. F. M., Marques, F. A. F., Fernandes, J. M., \& Cunha, J. P. S. (2011). A real time, wearable ECG and continuous blood pressure monitoring system for first responders. In Proceedings of 33rd Annual International Conference of the IEEE EMBS, Boston, Massachusetts USA (pp. 6894-6898). Piscataway, NJ: IEEE Press.

Soares, S. C., Esteves, F., Lundqvist, D., \& Öhman, A. (2009). Some animal specific fears are more specific than others: evidence from attention and emotion measures. Behaviour Research and Therapy, 47, 1032-1042. doi:10.1016/j.brat.2009.07.022

Soares, S. C., Esteves, F., \& Lundqvist, D. (2015). In the grip of fear: Dissociations in attentional processing of animal fearful individuals. Scandinavian Journal of Psychology, 56, 11-17. doi:10.1111/sjop. 12179

Stopczynski, A., Stahlhut, C., Petersen, M. K., Larsen, J. E., Jensen, C. F., Ivanova, M. G., . . . Hansen, L. K. (2014). Smartphones as pocketable labs: Visions for mobile brain imaging and neurofeedback. International Journal of Psychophysiology, 91, 54-66.

Timby, B. K. (2009). Fundamental nursing skills and concepts (9th ed.). Philadelphia, PA: Lippincott Williams \& Wilkins.

Wilhelm, F. H., \& Grossman, P. (2010). Emotions beyond the laboratory: Theoretical fundaments, study, design, and analytic strategies for advanced ambulatory assessment. Biological Psychology, 84, 552569. doi:10.1016/j.biopsycho.2010.01.017 